FOLIA

Amazónica

Revista del Instituto de Investigaciones

de la Amazonía Peruana

\title{
EVIDENCIA PRELIMINAR DEL EFECTO BORDE EN ANFIBIOS DE LA RESERVA NACIONAL PUCACURO, AL NORTE DE LA AMAZONÍA PERUANA
}

\author{
Rommel R. ROJAS ${ }^{1}$, Pedro E. PÉREZ-PEÑA ${ }^{2}$ \\ 1 Laboratório de Evolução e genética Animal-LEGAL, Universidade Federal do Amazonas-UFAM, \\ Av. Gen. Rodrigo Octávio Jordão Ramos, 6200, CEP 69077-000, Manaus, AM, Brazil. \\ rrojaszamora@gmail.com \\ 2 Instituto de Investigaciones de la Amazonía Peruana (IIAP), Programa de Investigaciones en \\ Biodiversidad Amazónica. Av. Abelardo Quiñones km 2.5, lquitos, Perú
}

\section{RESUMEN}

Los anfibios son organismos que poseen una sensible relación con la degradación de hábitats y pueden ser usados como bioindicadores. En este trabajo usamos datos preliminares de una evaluación rápida en la Reserva Nacional Pucacuro, Perú, para probar la influencia del efecto borde en anfibios e identificar potenciales especies indicadoras. Calculamos y comparamos estadísticamente la abundancia, riqueza, tamaños y fluctuaciones espaciales en especies de anfibios en tres zonas de muestreo a tres distancias (Borde, 100 y 200 metros) de áreas deforestadas. Nuestros resultados sugieren la existencia de diferencias en abundancia, riqueza y fluctuaciones espaciales de anfibios en relación con las distancias y sugiere un efecto borde al menos hasta los 100 metros de un área deforestada. Como potenciales especies indicadoras fueron identificadas Rhinella margaritifera, Chiasmocleis bassleri y especies de Pristimantis. A pesar de tratarse de una evaluación rápida, nuestros datos sugieren una fuerte tendencia de cambios espaciales en la riqueza y abundancia de anfibios a diferentes distancias de un área deforestada.

PALABRAS CLAVE: Amazonía, anuros, conservación, Loreto, restauración 


\title{
PRELIMINARY EVIDENCE OF THE EDGE EFFECT IN ANFHIBIANS OF THE PUCACURO NATIONAL RESERVE, NORTH OF THE PERUVIAN AMAZON
}

\begin{abstract}
Amphibians are organisms that have a sensitive relationship to the degradation of habitats and are used as habitat indicators. In this work we use preliminary data from a rapid assessment in the Pucacuro national reserve, Peru to test the influence of the edge effect on amphibians and identify potential habitat indicator species. We calculated and statistically compared the abundance, richness, sizes and spatial fluctuations in amphibian species of three sampling zones in three distances (Borde, 100 and $200 \mathrm{~m}$ ) of deforested areas. Our results suggest the existence of differences in abundance, richness and spatial fluctuations of amphibians in relation to distances and suggest edge effect at least $100 \mathrm{~m}$ from deforested area. As potential habitat indicator were identified Rhinella margaritifera, Chiasmocleis bassleri and species of Pristimantis. Despite being a rapid assessment, our data suggest a strong tendency for spatial changes in abundance of amphibians at different distances from a deforested area.
\end{abstract}

KEYWORDS: Amazonia, anurans, conservation, Loreto, restoration 


\section{INTRODUCCIÓN}

La Amazonía es una de las más ricas formaciones de vida en la tierra (Myers et al., 2000). Sin embargo, está sufriendo un gran impacto ambiental causado principalmente por la destrucción y fragmentación de hábitats, la ocupación de tierras para monocultivos y el establecimiento y expansión de asentamientos humanos (Fearnside, 2003).

Los anfibios están sufriendo una drástica declinación de sus poblaciones a nivel global (Collins, 2010). Situación causada principalmente por la destrucción de sus hábitats y el hongo quitridio (Batrachochytrium dendrobatidis) que están diezmando poblaciones andinas y amazónicas (Valencia-Aguilar et al., 2015; Becker et al., 2016). Los anfibios son organismos estrechamente relacionados con la calidad de hábitats pues poseen fases de vida acuática y terrestre, y una piel permeable sensible a sutiles cambios ambientales, esta condición los convierte en excelentes bioindicadores (Heyer et al., 1973; Lips et al., 2005; Souza et al., 2008).

La interacción entre los factores bióticos y abióticos es de suma importancia para el entendimiento de la dinámica forestal, y el efecto de borde es un ejemplo de estos cambios en el espacio físico y orgánico (Ramos, 2012). El efecto borde posee fuerte influencia en procesos ecológicos de paisajes fragmentados, siendo reconocidos tres niveles (Murcia, 1995): cambios en las condiciones ambientales, cambios en la abundancia y distribución de especies y cambios en las interacciones entre las especies. La influencia del efecto borde varía conforme su distancia a la zona deforestada, afectando las características ambientales en áreas más próximas a ella (Connel \& Slatyer, 1977; Ewers et al., 2007). De esta manera, el entendimiento de cómo las características ecológias cambian en las proximidades del borde son importantes para la comprensión de la dinámica de deforestación y su impacto en seres vivos (Metzger, 1998; Lindenmayer \& Fischer, 2007). La evaluación de la influencia del efecto borde en sus diferentes niveles permite evaluar la estabilidad e integridad biológica de ecosistemas degradados y permite reconocer especies indicadoras de la calidad de hábitat. Sin embargo, la influencia del efecto borde en paisajes amazónicos ha sido poco estudiada en la Amazonía.

En este trabajo mostramos evidencia de la influencia del efecto borde en comunidades de anfibios en la Reserva Nacional Pucacuro, ubicada al norte de la Amazonía peruana y, al mismo tiempo, identificamos potenciales especies indicadoras de calidad de hábitat.

\section{MATERIALES Y MÉTODOS}

\section{ÁREA DE ESTUDIO}

El estudio se llevó a cabo durante 12 días (1623 de Junio 2014) en bosques de terraza alta, hábitat no inundable con relieve disectado en la Reserva Nacional Pucacuro ubicada en el río del mismo nombre, afluente del río Tigre por la margen izquierda; situada en el Distrito del Tigre, Provincia de Loreto, Departamento de Loreto. La cuenca del río Pucacuro está en un bosque húmedo tropical, la temperatura media anual fluctúa entre $25.2-25.7^{\circ} \mathrm{C}$. La precipitación promedio anual fluctúa entre 2300 y $2900 \mathrm{~mm}$. El nivel del río es mayor entre los meses de febrero y agosto (época de creciente), mientras que entre los meses de agosto y diciembre el nivel del río es bajo pero con algunos periodos breves de creciente. Durante los meses de junio y julio, ocurren periodos breves de descenso moderado de la temperatura (que ocasionalmente llegan hasta $14 \mathrm{o} 15^{\circ} \mathrm{C}$ ), provocados por vientos del hemisferio sur (estación meteorológica del Curaray). 


\section{DISEÑO DEL MUESTREO}

La influencia del efecto borde se evaluó mediante la categorización de tres zonas: Campamento, Helipuerto y Polvorín. Cada zona poseía un área deforestada. Se evaluaron tres distancias a partir de cada área deforestada en cada una de las zonas: Borde o 0 metros (m), $100 \mathrm{~m} \mathrm{y}$
$200 \mathrm{~m}$. Cada distancia fue muestreada por 1 hora durante el día y noche, obteniéndose un total de cuatro pseudoreplicas por distancia del área deforestada (Figura 1).

La búsqueda de anfibios fue realizada mediante Visual Encounter Survey (VES) (Crump \& Scott, 1994) durante tres horas en los horarios

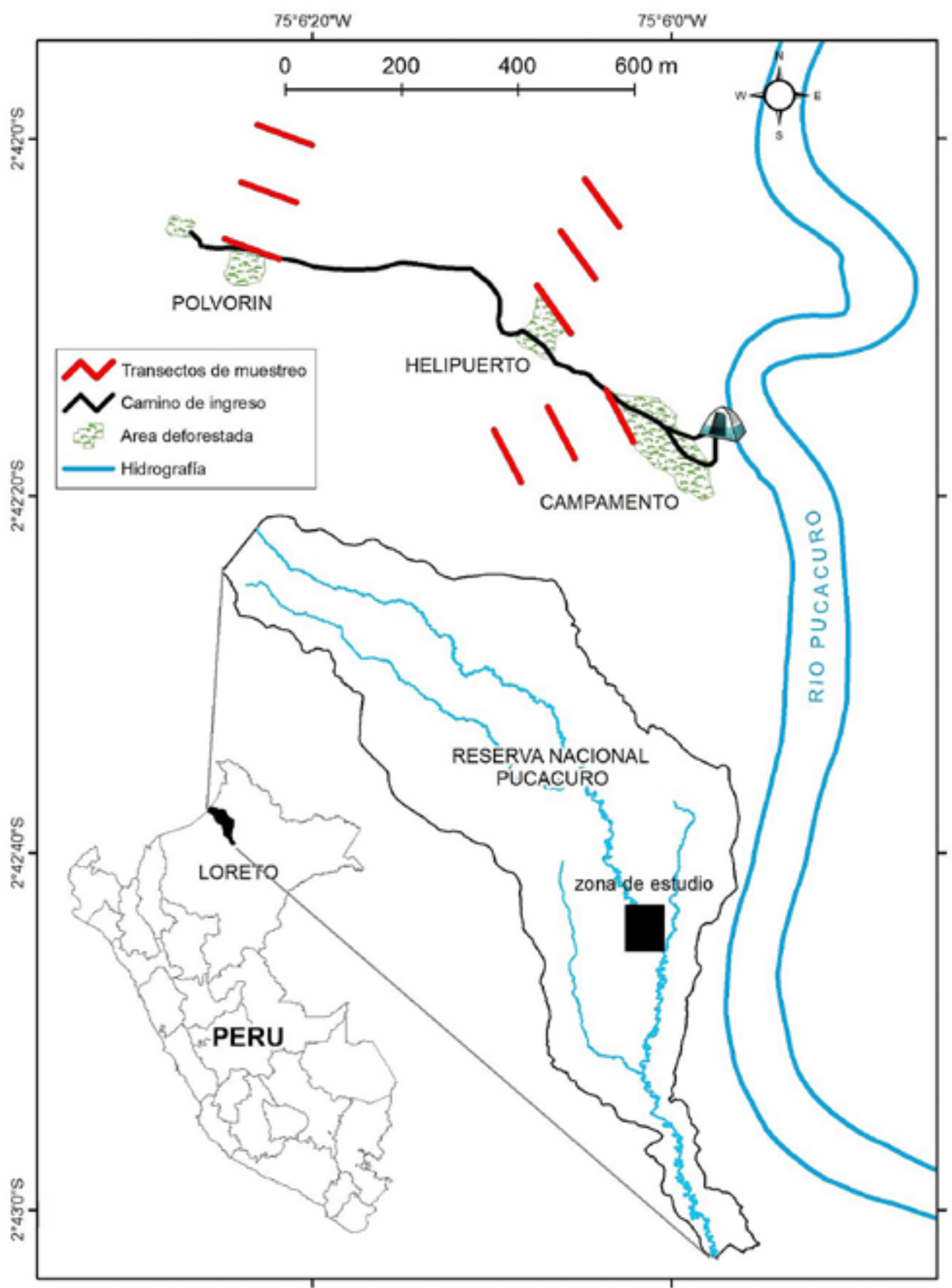

Figura 1. Diseño de muestreo para evaluación del efecto borde en anfíbios en la Reserva Nacional Pucacuro. de 8:00-11:00 y 19:00-23:00. Para analizar si el muestreo alcanzó la estabilidad (confiabilidad) de detección de especies fue realizada una curva de acumulación de especies (número de especies vs. días de muestreos).

\section{EFECTO BORDE EN ANFIBIOS}

Se realizó un análisis de Kruskal-Wallis para observar diferencias significativas entre la abundancia de anfibios en los tres puntos estudiados: Borde, 100 m y 200 m; seguidamente fue aplicada la prueba de comparación par a par de Mann-Whitney para identificar las distancias estadísticamente diferentes. Las diferencias entre la riqueza de anfibios por zonas $\mathrm{y}$ distancias fueron realizadas mediante la prueba de Kruskal Wallis. Para evaluar la influencia del efecto borde sobre los tamaños de anfibios, realizamos la siguiente categorización: anuros grandes $(<41 \mathrm{~mm})$, medianos $(\leq 21 \mathrm{~mm})$ y pequeños $(>20$ $\mathrm{mm}$ ). La biodiversidad $\beta$ se evaluó en términos de similitud 
usando análisis de agrupamiento con el índice de Bray Curtis. Para este análisis fue incluida una zona control denominada "descampado" (llamada así por ser una zona en proceso de restauración). Todos los análisis fueron realizados utilizando el software Past v.3.0 (Hammer et al., 2001).

\section{ESPECIES INDICADORAS}

Seguimos las características propuestas por Rice et al. (2012) donde señala que: las especies indicadoras de calidad de hábitat tienen que ser abundantes, conocidas taxonómicamente, activas en horas predecibles y cuya densidad cambie a lo largo de gradientes ambientales. Para identificar las especies indicadoras se seleccionaron las más abundantes. Se agruparon todos los individuos de la familia Craugastoridae (excepto Oreobates quixensis) por ser una familia de especies reconocidas como indicadoras y por presentar una mayor cantidad de especies en las distancias evaluadas. Seguidamente se realizó un análisis de Kruskal-Wallis para observar diferencias significativas entre la abundancia de las especies indicadoras en las tres distancias evaluadas (Borde, $100 \mathrm{~m}$ y $200 \mathrm{~m}$ ). Las comparaciones entre puntos se realizaron con la prueba MannWhitney. Todos los análisis fueron realizados utilizando el software Past v.3.0 (Hammer et al., 2001).

Se calculó el índice de abundancia (ind/ hora-hombre) por ser un estimador que esta relacionado con el tamaño poblacional y su precisión y exactitud depende del esfuerzo de muestreo. El análisis de fluctuación en la composición de especies por distancia del borde utilizó la curva de Whittaker. La dominancia de especies fue calculada por zona y por distancia del borde $(0 \mathrm{~m}, 100 \mathrm{~m}$ y $200 \mathrm{~m})$. Este cálculo fue realizado mediante el índice de abundancia (número de individuos/kilómetros recorridos).

\section{RESULTADOS}

El registro de las especies alcanzó el 83\% a los 12 días de muestreos (Tabla 1), el cual indica que casi todas las especies existentes en el área de estudio fueron detectadas. Se registraron un total de 30 especies pertenecientes a la clase Anfibia. Fueron encontrados dos órdenes: Anura y Caudata. El orden Anura presentó 06 familias, 12 géneros y 29 especies, mientras que el orden Caudata tuvo una familia, un género y una especie. En el orden Anura, la familia de mayor riqueza fue Craugastoridae (13 especies), seguido por la familia Hylidae (7 especies) y Leptodactylidae (4 especies); mientras que la familia con menor riqueza fue Aromobatidae (1 especie). Dentro del orden Caudata fue registrada sólo una especie: Bolitoglossa peruviana.

Tabla 1. Estimación de riqueza esperada y porcentaje de especies registradas durante 12 días de muestreos.

\begin{tabular}{|l|c|}
\hline Estimadores & Número de especies \\
\hline Chao1 & 35.33 \\
\hline Chao2 & 38.33 \\
\hline Chao y Lee1 & 31.09 \\
\hline Chao y Lee2 & 31.09 \\
\hline Jacknife1 & 38.89 \\
\hline Jacknife2 & 42.58 \\
\hline Bootstrap & 34.39 \\
\hline Michaelis-Menten & 38.30 \\
\hline Riqueza esperada $(\overline{\mathrm{X}} \pm$ SD $)$ & $36.00 \pm 4.02$ \\
\hline Riqueza observada & 30.00 \\
\hline \% especies registradas $\overline{\mathrm{X}}$ (rango) & $\mathbf{8 3 . 3 3}$ (71-97) \\
\hline
\end{tabular}




\section{EFECTO BORDE EN ANFIBIOS}

La riqueza de los anfibios por distancia al área deforestada indicó diferencias significativas entre la riqueza de especies en cada distancia evaluada (Borde, $100 \mathrm{~m}$ y $200 \mathrm{~m}$ ), $(p=0.044)$ (Tabla 2). Sin embargo, no mostró diferencias significativas entre la riqueza de anfibios por zona de muestreo (campamento, helipuerto y polvorín) ( $p \geq 0.05)$.

La abundancia total de anfibios presentó diferencias significativas $(p=0.05)$ entre todas las zonas (campamento, helipuerto y polvorín) $\mathrm{y}$ distancias (Borde, $100 \mathrm{~m} \mathrm{y}$ $200 \mathrm{~m}$ ) del área deforestada. Las comparaciones par a par de MannWhitney mostraron la existencia de diferencias significativas entre todos los puntos $(p \leq 0.05)$, excepto entre $100 \mathrm{~m}$ vs. $200 \mathrm{~m}$ ( $p=0.4502)$ (Tabla 3 ).

La influencia de la distancia del borde sobre el tamaño de los anfibios mostró diferencias significativas $(p \leq 0.05) \quad$ (Tabla 4). Anfibios pequeñosfueronmásabundantesenla distanciade 200 m, representando un 50,63\% de la población, mientras que fueron menos abundantes en el borde (7.59\%). Los anfibios medianos fueron detectados en mayor abundancia a $100 \mathrm{~m}$ del área deforestada (45.09\%) y menor abundancia en el borde (16.66\%). Anfibios grandes presentaron mayor abundancia a $200 \mathrm{~m} \mathrm{(51.06 \% )} \mathrm{de} \mathrm{la} \mathrm{zona}$ deforestada y menor abundancia en el borde (23.04\%).

La similitud existente entre las comunidades de anfibios en diferentes distancias de muestreos (descampado, borde, $100 \mathrm{~m}$ y $200 \mathrm{~m}$ ) indicó mayor semejanza entre las distancias $100 \mathrm{~m}$ y 200 m siendo más próximas a la distancia borde, mientras que el descampado presentó menor similitud con las otras distancias (Figura 2). Este patrón de similitud fue compartido en todas las zonas evaluadas.

\section{ESPECIES INDICADORAS}

Basados en las características propuestas por Rice et al. (2012) fueron seleccionadas las especies Chiasmoscleis bassleri, Rhinella margaritifera, Oreobates quixensis y todas especies de la familia Craugastoridae (por representar la familia de mayor riqueza).

La abundancia de Oreobates quixensis no mostró un patrón de disminución desde el interior al borde, al parecer su abundancia está condicionada a otros factores. Chiasmocleis bassleri y los Pristimantis mostraron diferencias y tuvieron abundancias muy reducidas en el borde. La especie $R$. margaritifera mostró diferencias en las tres distancias muestreadas, y fue la única 

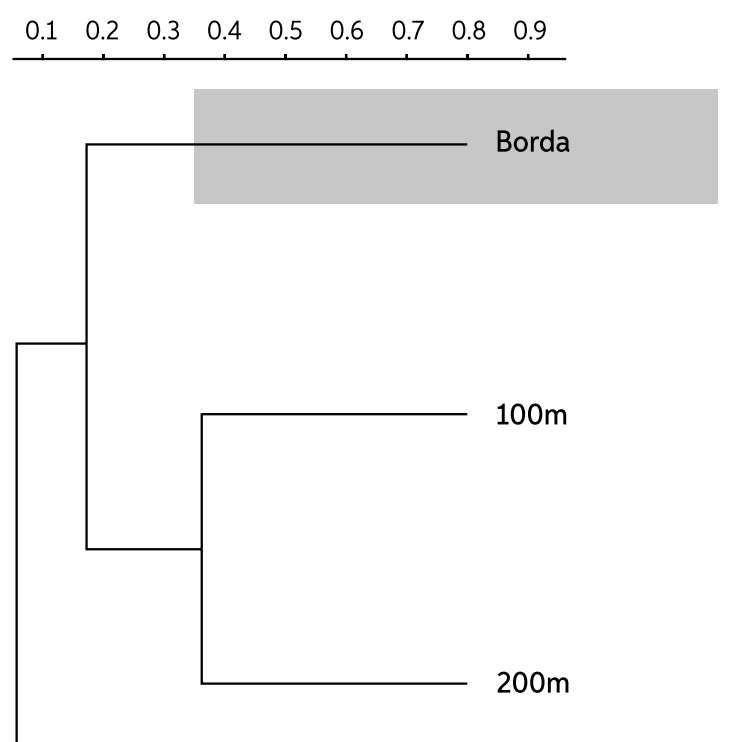

Descampado
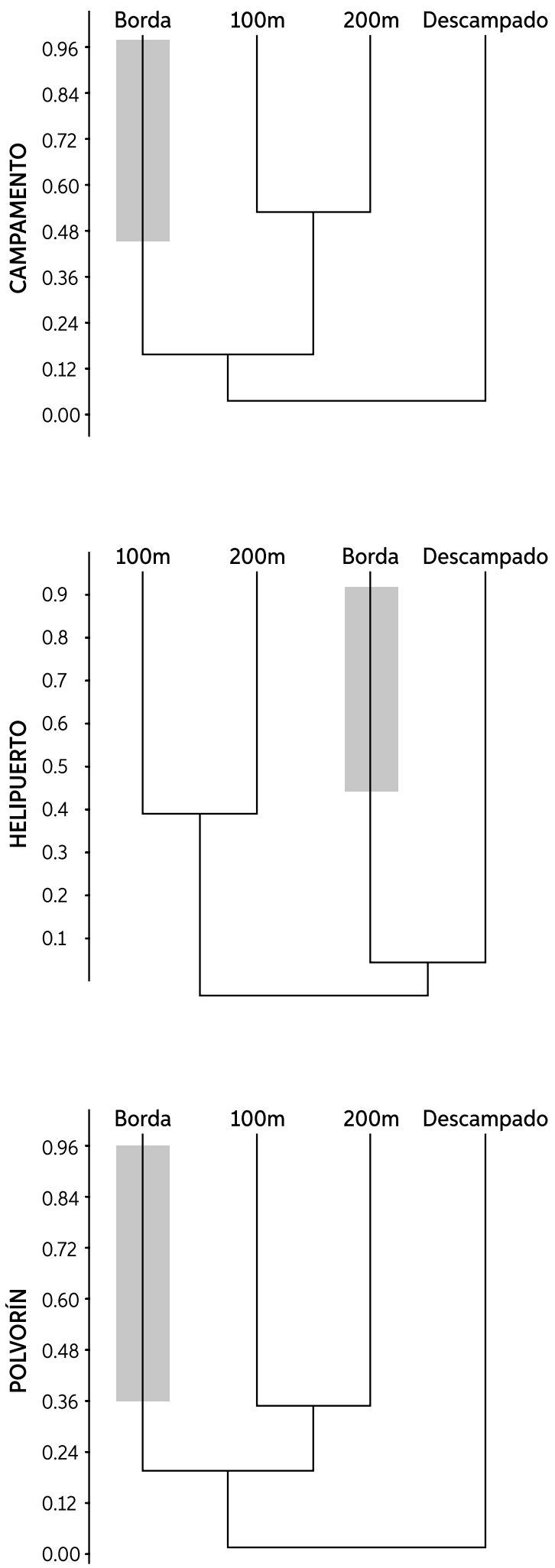

Figura 2. Análisis de similitud de Bray Curtis por zonas de muestreo de asambleas de anfíbios en diferentes distancias de un área deforestada. En este análisis la zona de Descampado fue incluido como zona control. 


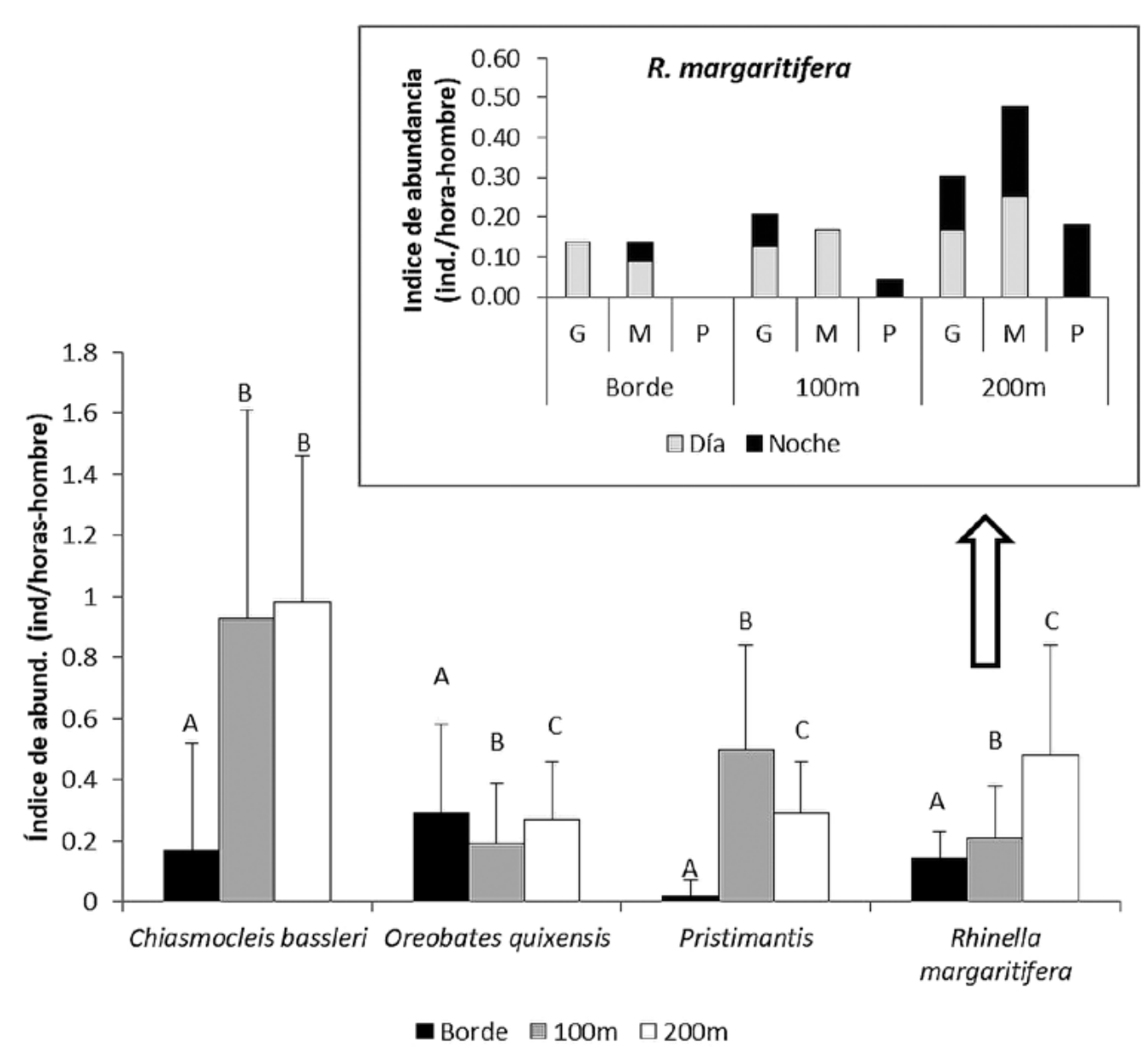

Figura 3. Abundancia de las especies más dominantes en la zona de estudio, las diferentes letras en cada par de barras indican diferencia significativa $(p<0.05)$. La figura interior indica la abundancia de los tamaños $(G=$ grande, $M=$ mediano, $P=$ pequeño) de Rhinella margaritifera durante el día y la noche en las tres distancia de muestreo.

que disminuyó gradualmente desde los $200 \mathrm{~m}$ hasta el borde (Figura 3). Es decir, las especies $R$. margaritifera, C. bassleri y las especies de Pristimantis fueron afectadas por el efecto borde, y pueden ser consideradas indicadoras de la perturbación del bosque. El análisis de abundancia por tamaño durante el día y noche de $R$. margaritifera, especie con disminución gradual, indicó que en el borde no hay individuos pequeños, sólo se registró individuos grandes y medianos en mayor proporción durante el día. $\mathrm{Al}$ interior del bosque, a $100 \mathrm{~m}$, se registró poca abundancia $(0.04$ ind./hora-hombre) de individuos pequeños y sólo durante la noche, mientras que a $200 \mathrm{~m}$ se registró mayor cantidad de pequeños (0.18 ind./ hora-hombre) pero sólo en la noche, además se registró similar abundancia durante el día y la noche en individuos medianos $(0.25$ y 0.23 ind./horas-hombre) y grandes $(0.17$ y 0.14 ind./hora-hombre).

La curva de Whittaker mostró diferencias en el número de especies dominantes en las tres distancias. $\mathrm{Si}$ se considera como especies dominantes a aquellas mayores a 0.2 ind./horahombre, en el borde se registró tres especies: Oreobates quixensis, Chiasmocleis bassleri y Rhinella margaritifera con 0.38, 0.29 y 0.25 ind./ hora-hombre. Al interior del bosque a los 100 $\mathrm{m}$, las especies dominantes fueron C. bassleri, $O$. quixensis, $R$. margaritifera y Adenomera andreae con $1.29 ; 0.29 ; 0.29 ; 0.29$ y 0.17 ind/ hora-hombre. La especie más dominante, $C$. bassleri, fue cuatro veces más abundante que $O$. quixensis, que fue la especie dominante del borde, además hubo una especie dominante más. Más al interior del bosque, a los $200 \mathrm{~m}$, hubo cinco especies dominantes: Chiasmocleis carvalhoi que 
índice de abundancia (ind./hora-hombre)

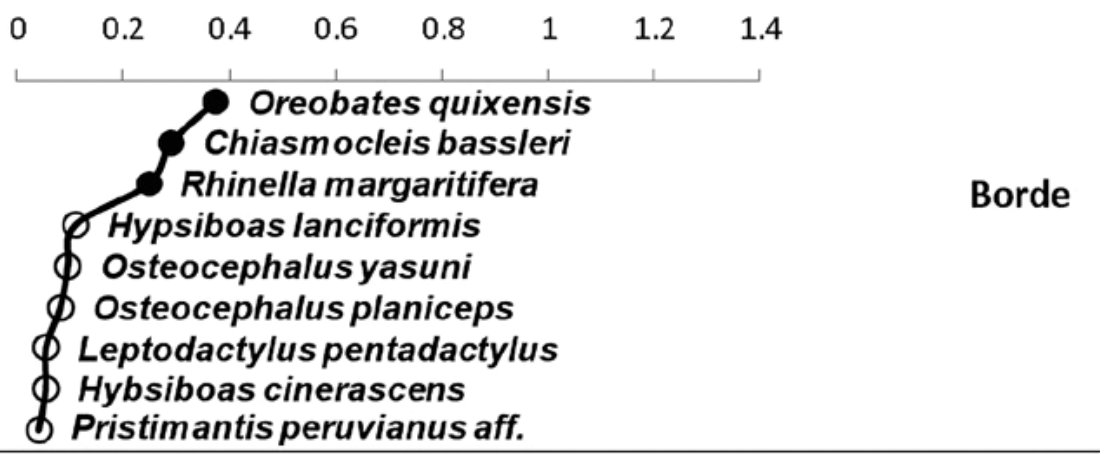

Preobates quixensis
$\begin{aligned} & \text { Rhinella margaritifera } \\ & \text { Adenom era andreae }\end{aligned}$
Chiasmocleis bassleri
$\oint_{\text {Pristimantis malkini }}$ Bolitoglossa peruviana
Pristimantis luscombei
Strabomantis sulcatus
Leptodactylus rhodomystax
Chiasmocleis magnova
Phyllomedusa vaillanti
Pristimantis ockendeni
Pristimantis ockendeni aff
Pristimantis lanthanites
Hypsiboas lanciformis
Osteocephalus deridens
Pristimantis delius
Pristimantis peruvianus
Pristimantis sp marron
Pristimantis sp verde

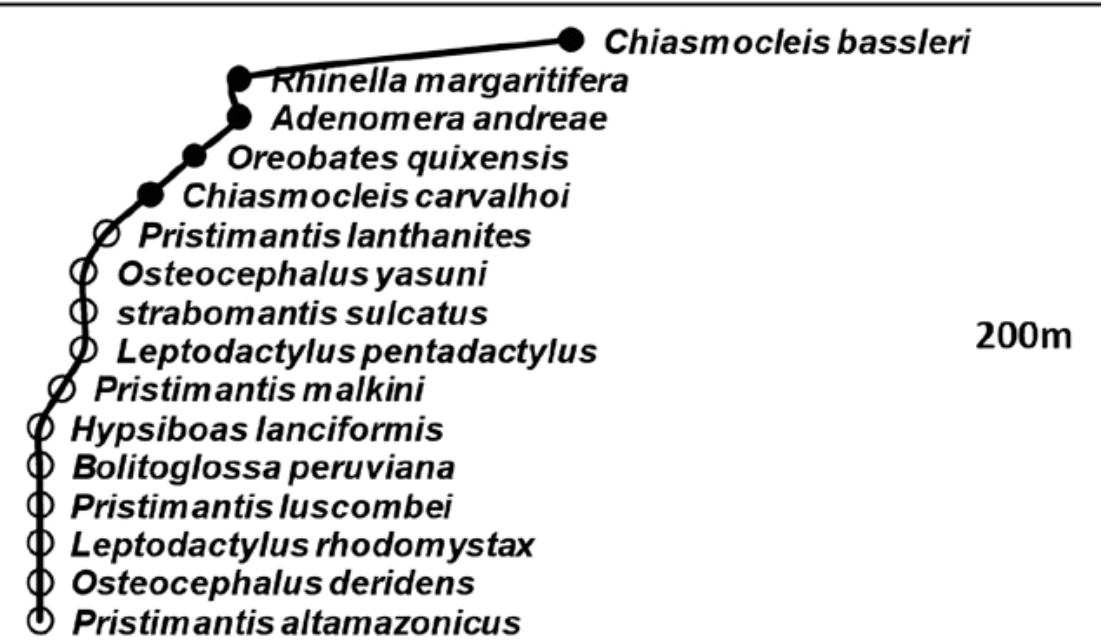

Figura 4. Curva de Whittaker utilizando la abundancia nocturna en diferentes distancias (Borde, 100m y 200m) desde un área deforestada. Los círculos negros indican las especies dominantes. estuvo ausente en el borde pero fue abundante tanto a los 100 y $200 \mathrm{~m}$, aunque esta abundancia no fue muy marcada como en las especies antes mencionadas (Figura 4).

\section{DISCUSIÓN}

A pesar de los pocos días de muestreo nuestra curva de acumulación de especies se estabilizó, indicando que la colecta de los datos fue suficiente para los análisis. Este tipo de evaluaciones rápidas son comunes en la Amazonía peruana y están relacionadas a disponibilidad logística (transporte, alimentación, disponibilidad de investigadores y colaboración interinstitucional). Así mismo, nuestro diseño de muestreo mostró ser adecuado para la evaluación de la influencia del efecto borde en anfibios de la Reserva Nacional Pucacuro y podría ser reproducido para otros lugares de nuestra Amazonía.

La influencia del efecto borde en anfibios fue reportado en otros trabajos (Rosenfied et al., 1992; De Maynadier \& Hunter, 1997; OsornaMuñoz, 1999; Ramos, 2012), estos estudios concuerdan con nuestros resultados, sugiriendo que las alteraciones micro-climáticas en condiciones y recursos ecológicos afectarían directamente la abundancia y riqueza de anfibios por causa de sus características biológicas (Herrera et al., 2004; Azevedo-Ramos \& De Carvalho, 2005; Lips et al., 2005; Bernarde, 2007). 
Nuestros resultados evidenciaron fluctuaciones espaciales (cada 100 metros del área deforestada) en abundancia y riqueza de anfibios, los cuales se vieron afectados a cada distancia evaluada (Borde, $100 \mathrm{~m}$ y $200 \mathrm{~m}$ ). Sin embargo, la ausencia de diferencias en la abundancia entre las distancias de $100 \mathrm{~m} \mathrm{vs}$. $200 \mathrm{~m}$ podría estar indicando bajos niveles de perturbación en estas distancias en relación con la distancia del área deforestada y al mismo tiempo mayor similitud de hábitat en distancias más alejadas (ver Figura 2). Estudios similares sugieren que la influencia del efecto borde puede llegar hasta unos 60 metros (Osorna-Muños, 1999) del área deforestada, mientras que otros indican que puede ser entre 25-200 m (De Maynaider \& Hunter, 1997). Basados en nuestros resultados sugerimos que la influencia del efecto borde estaría afectando la abundancia y riqueza de anfibios al menos dentro de los primeros $100 \mathrm{~m}$ del área deforestada.

Nuestros análisis demostraron que el efecto borde tuvo mayor impacto en especies de anfibios de tamaños medianos y especialmente en los pequeños (ver Tabla 4); siendo estas especies las que mostraron mayor potencial para representar los indicadores de la calidad de hábitat. Por otro lado, especies de grandes Tabla 4. Prueba de Kruskal Wallis por tamaños de anfíbios anuros en relación a su distribución en las distancias evaluadas.

\begin{tabular}{|l|c|c|c|c|}
\hline \multicolumn{1}{|c|}{ Tamaños } & Borde & $\mathbf{1 0 0 m}$ & $\mathbf{2 0 0 m}$ & p-valor \\
\hline Anuros grandes $(>41 \mathrm{~mm})$ & 11 & 12 & 24 & 0.0354 \\
\hline Anuros medianos $(21-40 \mathrm{~mm})$ & 17 & 46 & 39 & 0.0012 \\
\hline Anuros pequeños $(<20 \mathrm{~mm})$ & 6 & 33 & 40 & 0.0001 \\
\hline
\end{tabular}

2003; Tsuji-Nishikido \& Menin, 2011). De esta manera, la presencia o ausencia de estas especies en relación a la distancia del área deforestada estaría influenciada por la tolerancia fisiológica de cada especie a los cambios bióticos y abióticos y su capacidad de adaptación en ambientes perturbados.

El abundante número de individuos detectados y disminución al borde de Rhinella margaritifera, Chiasmocleis bassleriy las especies de Pristimantis sugieren que estas especies podrían representar indicadoras de calidad de hábitat. Entre estas, Rhinella margaritifera puede ser la mejor por tener disminución gradual, fácil de identificar y capturar, además la presencia de individuos pequeños indicaría hábitats mejor conservados. Esta especie es muy común en la Amazonía peruana, López (2009) y Pérez \& Yáñez (2003) sugieren a $R$. margaritifera como una especie de alta probabilidad de registro en los muestreos herpetológicos en Loreto. tamaños no representaron potenciales especies indicadoras, siendo más abundantes a partir de $100 \mathrm{~m}$ del área deforestada.

A pesar de no conseguir medir las variables ambientales dentro áreas estudiadas, diversos factores ambientales tales como cobertura vegetal, estructura de la vegetación, tipo de suelo, tamaño del fragmento deforestado, profundidad de la hojarasca entre otros (ver Pickett \& White, 1985), pudieron influenciar significativamente el patrón de distribución espacial y actividad de los anfibios (Souza et al., 2008; Gimenes \& Anjos, 


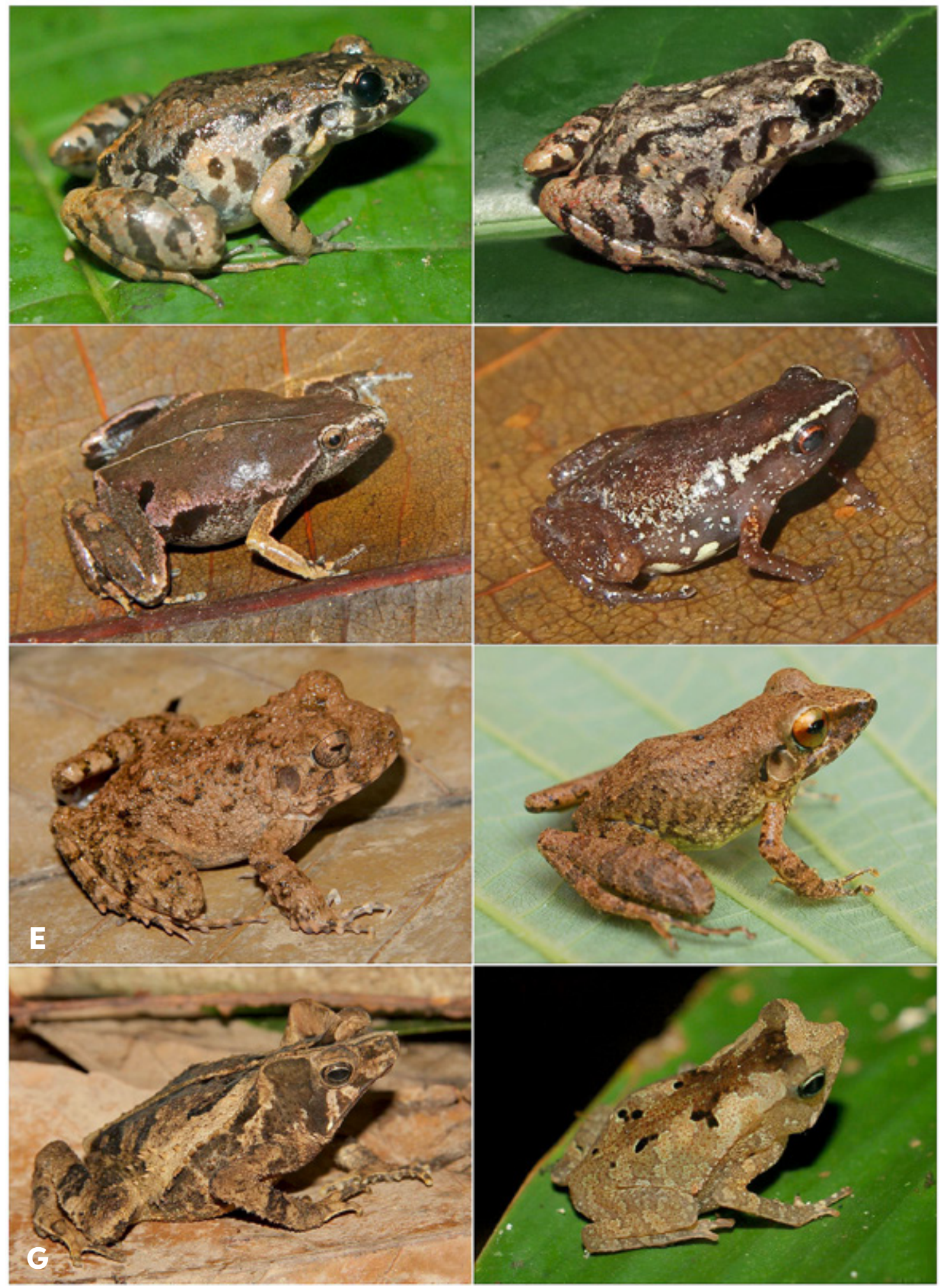

Figura 5. Especies más abundantes. Adenomera andreae hembra (A) y macho (B), Chiasmocleis bassleri (C), Chiasmocleis carvalhoi (D), Oreobates quixensis (E), Pristimantis malkini (F), Rhinella margaritifera hembra adulta $(G)$ y juvenil $(H)$. Fotos: Rommel Rojas (C, D, E, G), Pedro Pérez-Peña (A, F, H), Ehiko Ríos (B). 
su densidad cambia a lo largo de un gradiente de perturbación, la especie $C$. bassleri y especies de Pristimantis podrían también ser consideradas como anfibios indicadores de calidad de hábitat en la Reserva Nacional Pucacuro (Figura 5). Sin embargo, estos resultados se limitan en detectar especies indicadoras en ambientes terrestres por conocerse la gran diferencia en comunidades de anfibios acuáticos (Menin et al., 2007).

Es imprescindible continuar con evaluaciones biológicas dentro de hábitats en proceso de recuperación, para conocer detalladamente el fenómeno de efecto de borde en anfibios y otros grupos de animales y poder entender la dinámica de restauración de los bosques Amazónicos y su influencia en diversos grupos de organismos.

\section{BIBLIOGRAFIA CITADA}

Azevedo-Ramos, C.; de Carvalho, Jr.; Nasi, R. 2005. Animais como indicadores. Uma ferramenta para acessar a integridade biológica a pós a exploração madeireira em florestas tropicais. Brasilia. 62 pp.

Becker, C.; Rodrigues, D.; Lambertini, C.; Toledo, F.; Haddad, C. 2016. Historical dynamics of Batrachochytrium dendrobatidis in Amazonia. Ecography, 39:954-960.

Bernarde, P.S. 2007. Ambientes e temporada de vocalização da anurofauna no município de Espigão do Oeste, Rondônia, sudoeste da Amazônia, Brasil (Amphibia: anura). Biota Neotropica, 7: 87-92.

Buckland, S.T.; Anderson, D.; Burnham, K.; Laake. J. 1993. Distance Sampling: Estimating the Abundance of Biological Populations. Londres.

Collins, JP. 2010. Amphibian decline and extinction: what we know and what we need to learn. Diseases of Aquatic Organism, 92: 93-99. Connel, J.H.; Slatyer, R.O. 1977. Mechanisms of sucession in natural communities and their role in community stability and organization. American Naturalist, 111: 1119-1144.

Crump, M.; Scott Jr, N.J. 1994. Visual encounter surveys. In: Heyer RW, Donnelly MA, McDiarmid RA, Hayek LC, Foster MS, (Eds). Measuring and monitoring biological diversity: standard methods for amphibians. p. 84-92.

De Maynadier, P.; Hunter, M. 1995. The relationship between forest management and amphibian ecology: a review of the North American literature. Environmental Reviews 3: 230-261.

Ewers, R.M.; Thorpe, S.; Didham, R.K. 2007. Synergistic interactions between edge and area effects in a heavily fragmented landscape. Ecology, 88(1): 96-106.

Fearnside, P.M. 2003. A Floresta Amazônica nas Mudanças Globais. Instituto Nacional de Pesquisas da Amazônia-INPA, Manaus, Brasil.

Gimenes, L.; Anjos, L. 2003. Efeitos da fragmentação florestal sobre as comunidades de aves. Maringá, 25 (2): 391-402.

Hammer, Ø.; Harper, D.A.T.; Ryan, P.D. 2001. PAST: Paleontological statistics software package for education and data analysis. Palaeontologia Electronica, 4(1): 1-9.

Herrera-Montes, A.; Olaya, L.; Castro, F. 2004. Incidencia de la perturbación antrópica en la diversidad, la riqueza y la distribución de Eleutherodactylus (Anura:Leptodactylidae) en un bosque nublado del suroccidente colombiano. Caldasia, 26(1): 265-274.

Heyer, W.; Berven, K. 1973. Species diversities of herpetofaunal samples from similar microhabitats al two tropical sites. Ecology, 54: 642-645.

Lindenmayer, D; Fischer, J. 2007. Edge Effects. In: Lindenmayer, D.; Hobbs, R.J. (Eds). Managing and designing landscapes for conservation. $\mathrm{p}$. 165-178.

Lips, K.R.; Burrowes, P.A.; Mendelson, J.R.; ParraOlea, G. 2005. Amphibian population declines 
in Latin America: a synthesis. Biotropica 37(2):222-226.

Lopes, J. 2009. Herpetofauna en bosque de terraza media en el sector Km 34,5-36,5 de la carretera Bellavista-Mazan (Proyecto Amazonas), LoretoPerú. Tesis para optar por el título de Biólogo de la Facultad de Ciencias Biológicas-UNAP, 70 pp.

Lovich, R.E.; Hayes, W.; Mushinsky, J.; Rodda, G.H. 2012. Transect Surveys, including Line Distance. In: McDiarmid, W.; Foster, M.S.; Guyer, C.; Gibbons, W.; Chernof, N. (Eds). Reptile Biodiversity. Standard methods for inventory and monitoring. p. 227-234.

Menin, M.; Lima, A.P.; Magnusson, W.E.; Waldez, F. 2007. Topographic and edaphic effects on the distribution of terrestrially reproducing anurans in Central Amazonia: mesoscale spatial patterns. Journal of Tropical Ecology 17(2):86-91.

Metzger, J.P. 1998. Estrutura da paisagem e fragmentação: Análises bibliográfica. Anais da Academia Brasileira de Ciências, 71: 445-462.

Murcia, L. 1995. Edge effects in fragmented Forest: implications for conservation. Tree, 10: 58-62.

Myers, N.; Mittermeier, R.; Mittermeier, C.; da Fonseca; G.; Kent, J. 2000. Biodiversity hotspots for conservation priorities. Nature, 403: 853-858.

Osorna-Muñoz, M. 1999. Evaluacion del efecto de Borda en poblaciones de Eleutherodactylus viejas (Amphibia:Anura:Leptodactylidae), frente a los corredores de servidumbre en diferentes estados de regeneración, en dos bosques intervenidos por líneas de transición eléctrica de alta tensión. Revista Academica Colombiana de Ciencias, 13: 350-356.

Pérez, P., Yáñez, C. 2003. Inventario de Anfibios y Reptiles en el rio Pucacuro Loreto, Perú.
Tesis para optar por el título de Biólogo de la Facultad de Ciencias Biológicas-UNAP, 136 pp. Pickett, T. White, P. (1985). Patch dynamics: a synthesis, p.371-384. In: The Ecology of natural disturbance and patch dynamics. San Diego, Academic Press, 472 pp.

Ramos, L. 2012. Influência da borda sobre a anurofaua do Parque Estadual Carlos Botelho (SP). Tesis de post-grado Universidade de São Paulo, Escola superior de Agricultura "Luiz de Queiroz", São Paulo, Brasil. 50pp.

Rice, K.; Mazzotti, F.; Waddle, H.; Conil, M. 2002. Uso de Anfíbios como indicadores del éxito de la restauración de Ecosistemas. (https:// edis.ifas.ufl.edu/pdffiles/UW/UW23600.pdf). Acceso: 06/08/2018.

Rosenfield, R.; Morasky, J.; Bielefeldt, J.; Loope, W. 1992. Forest fragmentation and island biogeography: a summary and bibliography. Technical report U.S National Park Service. Washinton D.C. 198 pp.

Souza, V.; Souza, M.; Morato, M. 2008. Efeitos da sucessão florestal sobre a anurofauna (Amphibia:Anura) da Reserva Catuabba e seu entorno, Acre, Amazônia Sul-Oriental. Revista Brasileira de Zoologia, 25: 49-57.

Tsuji-Nishikido, B. M.; Menin, M. 2008. Distribuição de anuros em áreas ripárias de um fragmento florestal urbano na Amazônia Central. Biota Neotropica 11: 63-70.

Valencia-Aguilar, A.; Ruano-Fajardo, G.; Lambertini, C.; Leite D.D.S.; Toledo L.F.; Mott, T. 2015. Chytrid fungus acts as a generalist pathogen infecting species-rich amphibian family in Brazilian rainforests. Diseases of Aquatic Organisms, 114:61-67.

Zug, G., L. Vitt y J. Caldwell. (2001). Herpetology. An introductory biology of amphibians and reptiles. USA. $630 \mathrm{pp}$.

Recibido: 22 de marzo de 2018 Aceptado para publicación: 11 de junio de 2018 